\title{
Precision Core Temperature Measurement of Metals Using an Ultrasonic Phase-Shift Method
}

\author{
Olaide F. Olabode *(-), Simon Fletcher, Andrew P. Longstaff $(-$ and Naeem S. Mian \\ Center for Precision Technologies, University of Huddersfield, Huddersfield HD1 3DH, UK \\ * Correspondence: olaide.olabode@hud.ac.uk
}

Received: 29 June 2019; Accepted: 2 September 2019; Published: 4 September 2019

\begin{abstract}
Temperature measurement is one of the most important aspects of manufacturing. There have been many temperature measuring techniques applied for obtaining workpiece temperature in different types of manufacturing processes. The main limitations of conventional sensors have been the inability to indicate the core temperature of workpieces and the low accuracy that may result due to the harsh nature of some manufacturing environments. The speed of sound is dependent on the temperature of the material through which it passes. This relationship can be used to obtain the temperature of the material provided that the speed of sound can be reliably obtained. This paper investigates the feasibility of creating a cost-effective solution suitable for precision applications that require the ability to resolve a better than $0.5^{\circ} \mathrm{C}$ change in temperature with $\pm 1^{\circ} \mathrm{C}$ accuracy. To achieve these, simulations were performed in MATLAB using the k-wave toolbox to determine the most effective method. Based upon the simulation results, experiments were conducted using ultrasonic phase-shift method on a steel sample (type EN24T). The results show that the method gives reliable and repeatable readings. Based on the results from this paper, the same setup will be used in future work in the machining environment to determine the effect of the harsh environment on the phase-shift ultrasonic thermometry, in order to create a novel technique for in-process temperature measurement in subtractive manufacturing processes.
\end{abstract}

Keywords: core temperature measurement; phase-shift method; manufacturing; ultrasonic thermometry

\section{Introduction}

Dimensional accuracy and the surface integrity of manufactured products are key specifications that determine conformance to the design intent. The adherence to these specifications is an indication of the quality of the product and that it will meet the needs of customers. Temperature variation during manufacturing can influence both the dimensional accuracy and the surface integrity of the product. Therefore, temperature control or compensation of the thermal effects is essential in order to produce high quality components.

Exact temperature variation values are not given in the literature, as it is difficult to get a generic value due to different manufacturing processes. However, temperature variation in a workpiece during machining can reach $10{ }^{\circ} \mathrm{C}$. A $10^{\circ} \mathrm{C}$ variation will result in a differing expansion in different materials. In precision manufacturing, workpieces often need to be machined with a dimensional error of less than $5 \mu \mathrm{m}$. The accuracy and resolution with which workpiece temperature needs to be measured for different materials is given in Table 1. 
Table 1. Required accuracy and resolution for the precision manufacturing of different materials.

\begin{tabular}{ccccc}
\hline Material & $\begin{array}{c}\text { Coefficient of } \\
\text { Thermal } \\
\text { Expansion } \\
\left(\times \mathbf{1 0}^{-\mathbf{6}} /{ }^{\circ} \mathbf{C}\right)[1]\end{array}$ & $\begin{array}{c}\text { Expansion of 200 } \\
\text { mm Part at 10 } \\
\text { Change in } \\
\text { Temperature }(\boldsymbol{\mu m})\end{array}$ & $\begin{array}{c}\text { Required } \\
\text { Temperature } \\
\text { Accuracy }\left({ }^{\circ} \mathbf{C}\right)\end{array}$ & $\begin{array}{c}\text { Required } \\
\text { Temperature } \\
\text { Resolution }\left({ }^{\circ} \mathbf{C}\right)\end{array}$ \\
\hline Structural Steel & 12 & 24 & \pm 2 & 1 \\
Aluminum & 24 & 48 & \pm 1 & 0.5 \\
Cast Brass & 21 & 42 & \pm 1.2 & 0.6 \\
Tungsten & 4.6 & 9.2 & \pm 5.4 & 2.7 \\
Copper alloys & 18 & 36 & \pm 1.4 & 0.7 \\
\hline
\end{tabular}

The core temperature variation of metals affects their dimensional accuracy. The existing temperature measurement methods in manufacturing are limited in that they only represent the surface temperature of the workpiece. This paper investigates the possibility of creating a novel system for the precision core temperature measurement of metals which can be adapted for use in different manufacturing processes. One such manufacturing process is the machining process. Metal cutting is arguably the most important aspect of manufacturing and there have been advancements in optimizing the rate of metal cutting. New technologies have helped to increase the cutting speed, depth of cut and feed rate. However, with these increments also comes the increase in heat generation originating near the tool-workpiece interface [2]. Combined with effects from change in ambient temperature, heat sinking to fixtures and non-deterministic heat transfer between the component and cutting fluids, indirect monitoring of the temperature of the workpiece is a very challenging problem. Most of the temperature measurement techniques for manufacturing described in literature only deal with the machine [3] or cutting tool temperature [2].

One of the temperature measurement methods for workpieces reported in literature is the tool/workpiece thermocouple [4-6]. The main difficulties reported concerning the use of this method are the parasitic electromotive force (EMF) from secondary joints, the necessity for the accurate calibration of the tool and workpiece as a thermocouple pair, the need to isolate the thermocouple from the environment and the lack of clarity on what the EMF represents [5,6]. Also, this method does not indicate the core temperature of the workpiece. Infrared thermometry is another method that has been used in both dry conditions and with the presence of coolant [7]. However, the measurement only represents the surface temperature, and the accuracy that can be achieved with this method is less than the required accuracy for the precision machining of some materials, as infrared cameras' stated accuracy is typically $\pm 2{ }^{\circ} \mathrm{C}$ [8]. Moreover, this value is only valid in ideal conditions, as the accuracy will greatly reduce in harsh machining environments. Infrared cameras also require a good line of sight to work. Of all the methods previously used, none gives a direct indication of the core temperature of the workpiece, which is the parameter that affects the dimensional expansion.

The speed of sound in any material is dependent on the temperature of the medium of propagation. This dependence has been used in a variety of ways to measure the temperatures of different media. The pulse-echo technique uses the time-of-flight method to measure ultrasonic velocity which can then be related to the temperature of the medium [9]. The pulse-echo method is relatively simple, but the resolution of measurement may reduce with distance due to attenuation of the echo signal [10]. Another main technique is the continuous wave method which evaluates the distance of ultrasonic travel by computing the phase-shift between transmitted and received signals [11]. Some modifications and combinations of the two techniques have also been used, such as the two-frequency continuous wave method [11,12] and multiple frequency continuous wave method [13,14]. These techniques make use of two or more ultrasonic frequencies to increase the range of measurement and improve resolution.

Hu et al., using the temperature range of 25 to $200^{\circ} \mathrm{C}$, modified the ultrasonic velocity equation based on the effects thermal expansion has on the travel path of ultrasonic waves. They reported an increase in accuracy based on the compensations made for expansion [15]. Another recent work by Ihara et al. is the use of laser ultrasonic thermometry to measure the internal temperature of heated 
cylindrical rods. The authors reported that the results almost agree with those measured using a thermocouple, however the accuracy of the method is not sufficiently described [16]. Different methods of ultrasonic measurement have been used and modified for use in different fields of measurement, however, their use in precision manufacturing is not sufficiently described in the literature.

This paper explores the use of ultrasonic waves for the precision core temperature measurement of a steel workpiece. The results from simulation using the two main techniques are evaluated to find the best option for core temperature measurement in metals. The initial work consists of simulations in the k-Wave MATLAB toolbox-an open source toolbox for time domain acoustic and ultrasound simulations [17]. This was used to study the two main ultrasonic thermometry methods-the ultrasonic pulse-echo and phase-shift methods. $\mathrm{k}$-Wave was chosen for simulation because of its flexibility for defining different parameters and media of interest-it also gives a real-time A-scan of the propagation medium during simulation, as well as its propagation plot. In an A-scan, the amplitude of an ultrasonic pulse is represented as displacement in y-axis and the corresponding travel time is represented on the $x$-axis. Based on the simulation results, ultrasonic phase-shift experiments were conducted in a metrology laboratory and the results of these experiments will serve as input to a future experiment which involves the use of the developed techniques in subtractive manufacturing processes.

\section{Materials and Methods}

The Pulse-echo method is the traditional means of ultrasonic measurement. It uses the principle of time-of-flight (tof), where an ultrasonic pulse is propagated through a medium and the pulse is reflected when it encounters a medium of different physical property [18]. The tof from the ultrasonic transmitter to the receiver and the length of the travel path is used to compute the ultrasonic velocity [19]. This relationship is given as:

$$
c=\frac{d}{t o f}
$$

where $c$ is the ultrasonic velocity, $d$ is the distance travelled and tof is the time of flight [20].

The phase-shift method, on the other hand, uses the difference in the phase of steady state frequency ultrasonic wave between transmitted and received signals [13]. For an ultrasonic wave of known frequency travelling through a known distance, the phase difference between the transmitted and received signals can be used to compute the ultrasonic velocity through the medium. The relationship between the ultrasonic velocity and phase-shift is given in the equation below:

$$
L=\left(n+\frac{\varphi}{2 \pi}\right) \frac{c}{f}
$$

where $L$ is the distance between the transmitter and receiver, $n$ is the integer number of wave periods, $\phi$ is the phase-shift, $f$ is the ultrasonic frequency and $c$ is the ultrasonic velocity through the medium [19].

A modification of the phase-shift method which considerably improves both the range and resolution of measurement is the two-frequency continuous wave method (TFcw). It uses two frequencies for ultrasonic velocity computation. The TFcw equation is given as:

$$
c=\frac{2 \pi L \Delta f}{\Delta \varphi}
$$

where $c$ is the ultrasonic velocity, $L$ is the length of travel, $\Delta f$ is the difference between the two frequencies $\left(f_{1}-f_{2}\right)$ and $\Delta \phi$ is the difference between the phase-shifts given as [13]:

$$
\begin{gathered}
\Delta \varphi=\varphi_{1}-\varphi_{2}, \quad \text { if } \varphi_{1}>\varphi_{2}, \\
\Delta \varphi=\varphi_{1}+2 \pi-\varphi_{2}, \quad \text { if } \varphi_{1}<\varphi_{2}
\end{gathered}
$$


By adding a third frequency, the range and the resolution can both be further improved. This technique is known as the multiple frequency continuous wave method (MFcw) and the MFcw equation is given as [21]:

$$
c=\frac{L}{\operatorname{Int}\left[\frac{\Delta \varphi_{1}}{2 \pi} \frac{\Delta f_{2}}{\Delta f_{1}}\right] \frac{c}{\Delta f_{2}}+\operatorname{Int}\left[\frac{\Delta \varphi_{2}}{2 \pi} \frac{f_{1}}{\Delta f_{2}}\right] \frac{c}{f_{1}}+\frac{\varphi_{1}}{2 \pi} \frac{c}{f_{1}}}
$$

\subsection{Simulations}

Three simulations were performed in MATLAB R2017b using the k-Wave toolbox. The first simulation was set up to resolve $0.1^{\circ} \mathrm{C}$ change in temperature with tof of ultrasonic wave. Steel was chosen as the medium of propagation with a nominal length of $200 \mathrm{~mm}$. The k-Wave grid $(\mathrm{N} x)$ was defined as $6.561 \times 10^{3}$ grids, the spacing $(d x)$ was defined as $1.2 \times 10^{-4}$. The ultrasonic wave parameters were set up to achieve sensitivity of $0.1^{\circ} \mathrm{C}$. The sensor position for the simulation is given in Figure 1 .

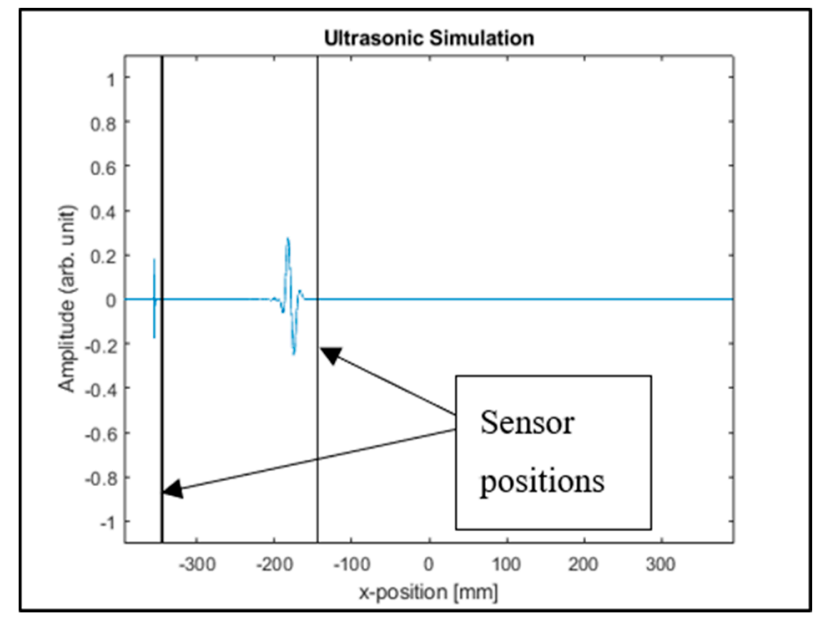

Figure 1. A-scan image of simulation.

The ultrasonic velocity used is based on the temperature-velocity relationship given by Ihara et al. [22]. This is given as:

$$
v(T)=-0.636 T+5917.6
$$

where $v(T)$ is the temperature-dependent ultrasonic velocity and $T$ is the temperature.

The simulation was run over the range of 25 to $25.5^{\circ} \mathrm{C}$ to observe if the corresponding change in time of flight can be reliably measured. Equation (6), which was used for the simulation, is almost linear for a temperature range of $0-200{ }^{\circ} \mathrm{C}$ [22] -hence, in this simulation, the sensitivity is prioritized over range. The peak detection technique was used to record the time the ultrasonic pulse strikes the sensor at both the transmitting and receiving positions [20].

The second simulation was carried out to observe the individual effect of change in ultrasonic velocity and change in material dimension (expansion) on tof. This was performed in order to verify if the tof can be reliably estimated from the change in velocity alone, expansion alone or by combining both. The ultrasonic velocity was varied using Equation (6), while material expansion was varied using Equation (7) given below:

$$
\Delta L=\alpha_{L} \Delta T L
$$

where $\Delta L$ is the change in length, $\alpha_{L}$ is the linear coefficient of thermal expansion, $\Delta T$ is the change in temperature and $L$ is the original material length.

The third simulation was performed using the phase-shift method, with the aim of obtaining the frequency pair which can consistently sense a $0.1^{\circ} \mathrm{C}$ change in temperature. This same simulation was modified for the MFcw method and, for this simulation, the length of material was scaled down by 
a factor of ten to reduce computational load. Also using the phase-shift method, a simulation was performed for a $15 \mathrm{~mm}$ piece of steel-this was performed to predict the possibility of using the $5 \mathrm{MHz}$ transducer to obtain a $10^{\circ} \mathrm{C}$ range and $0.1^{\circ} \mathrm{C}$ resolution.

\subsection{Simulation Results}

In order to achieve a measurement resolution of $0.1^{\circ} \mathrm{C}$, a tone burst of $1.2 \mathrm{MHz}$ and sampling frequency of $10 \mathrm{GHz}$ were used. The recorded tone burst at $25^{\circ} \mathrm{C}$ and the tof for the whole range of simulations are given in Figure 2 and Table 2 respectively.

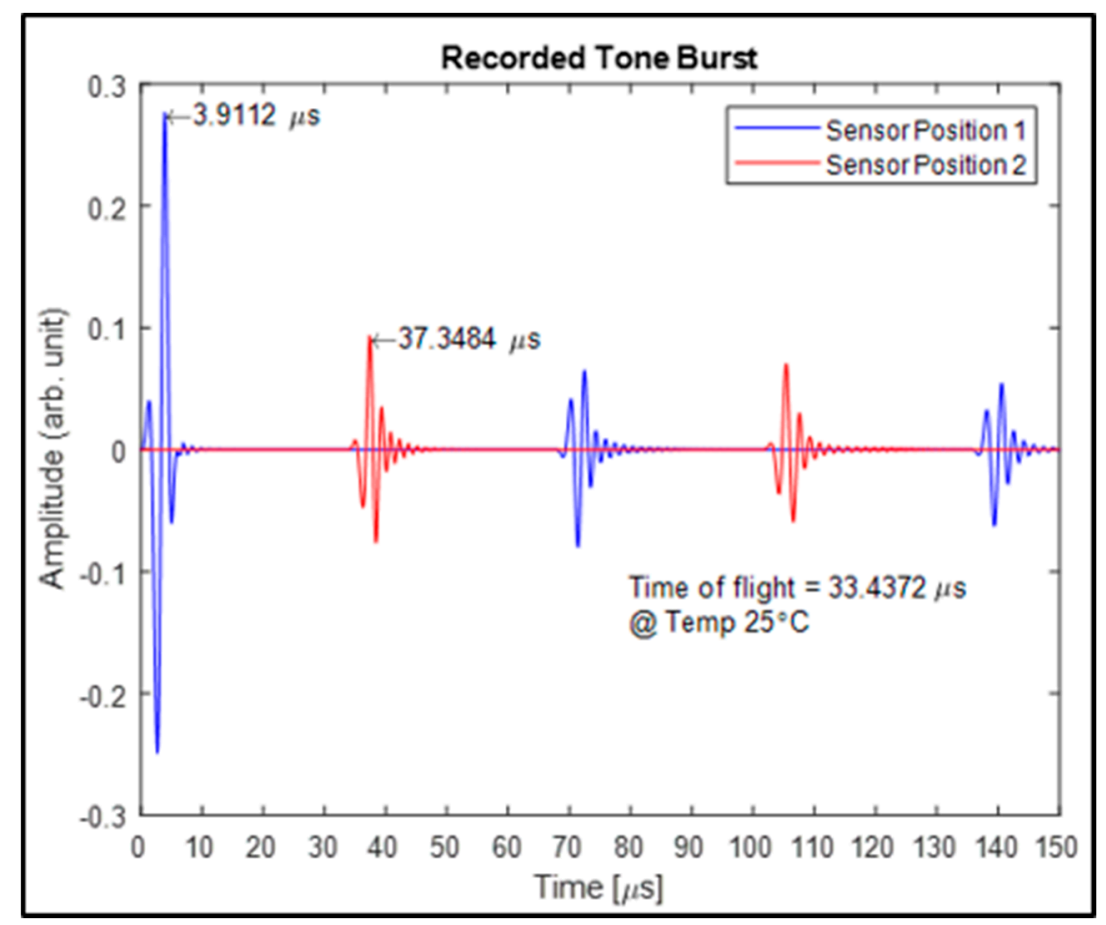

Figure 2. Recorded tone burst.

Table 2. Time of flight variation with temperature.

\begin{tabular}{ccc}
\hline Temperature $\left({ }^{\circ} \mathbf{C}\right)$ & Velocity $(\mathrm{m} / \mathbf{s})$ & Tof $(\mu \mathbf{s})$ \\
\hline 25.0 & 5901.70 & 33.4372 \\
25.1 & 5901.63 & 33.4376 \\
25.2 & 5901.57 & 33.4380 \\
25.3 & 5901.51 & 33.4384 \\
25.4 & 5901.45 & 33.4388 \\
25.5 & 5901.38 & 33.4392 \\
\hline
\end{tabular}

With $10 \mathrm{GHz}$ sampling frequency, a $0.1{ }^{\circ} \mathrm{C}$ change in temperature will cause a change in time of flight that can be resolved at the fourth decimal place of tof in microseconds. The costs of pulsers/receivers that sample at $10 \mathrm{GHz}$ frequency are considerably high.

From the findings of Thara et al., the relationship between ultrasonic velocity and temperature is almost linear within the range of 0 to $200{ }^{\circ} \mathrm{C}$ [22], as is the effect from the change in distance from thermal expansion. The simulations for expansion and ultrasonic speed were performed over this linear range. The result of this simulation is shown in Figure 3. 


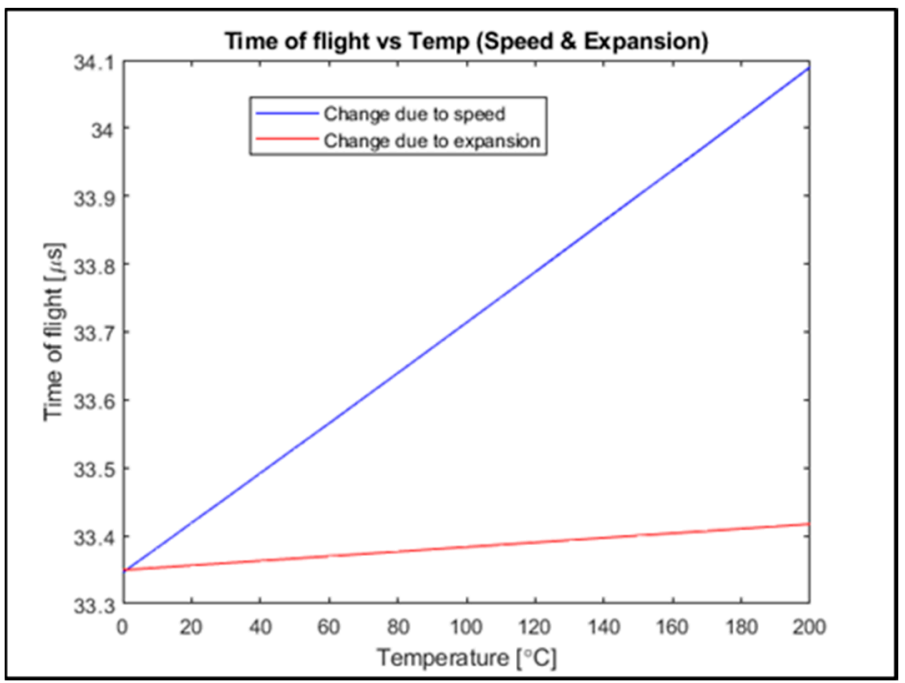

Figure 3. Effect of change in speed and expansion on tof.

Figure 3 shows that the time of flight varied largely due to changes in velocity, while the variation due to expansion is relatively smaller. Expansion across the range of 0 to $200{ }^{\circ} \mathrm{C}$ is $480 \mu \mathrm{m}$ in total for $200 \mathrm{~mm}$ steel. The tof can be reliably estimated by considering ultrasonic velocity while compensating for expansion.

The MFcw technique was used in the third simulation for the estimation of ultrasonic velocity. First, relatively low frequencies of 0.6 and $0.5 \mathrm{MHz}$ were used to estimate ultrasonic velocity through phase-shift—this is the TFcw technique. Thereafter, based on Equation (3), 0.5, 0.51 and $10 \mathrm{MHz}$ were used to improve the sensitivity of the simulation for a $0.1^{\circ} \mathrm{C}$ change in temperature (MFcw). The results for the simulations are given in the Figure $4 \mathrm{a}, \mathrm{b}$ and Table 3 respectively.

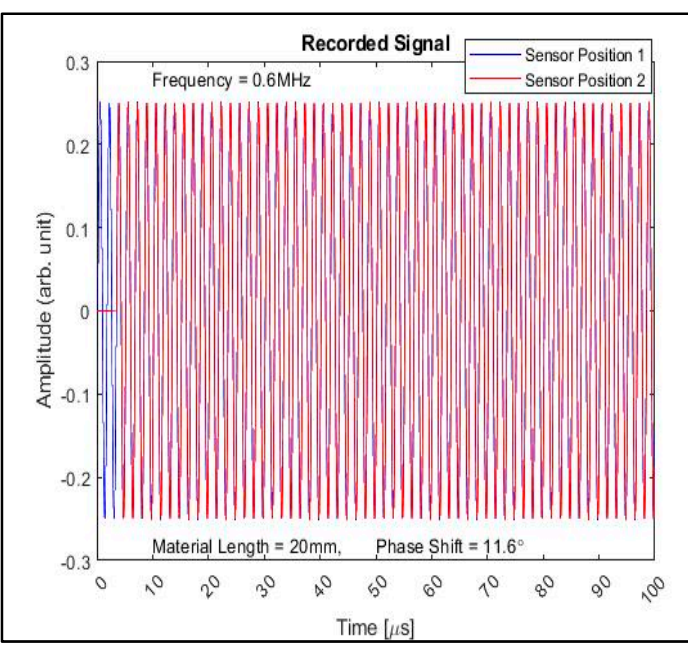

(a)

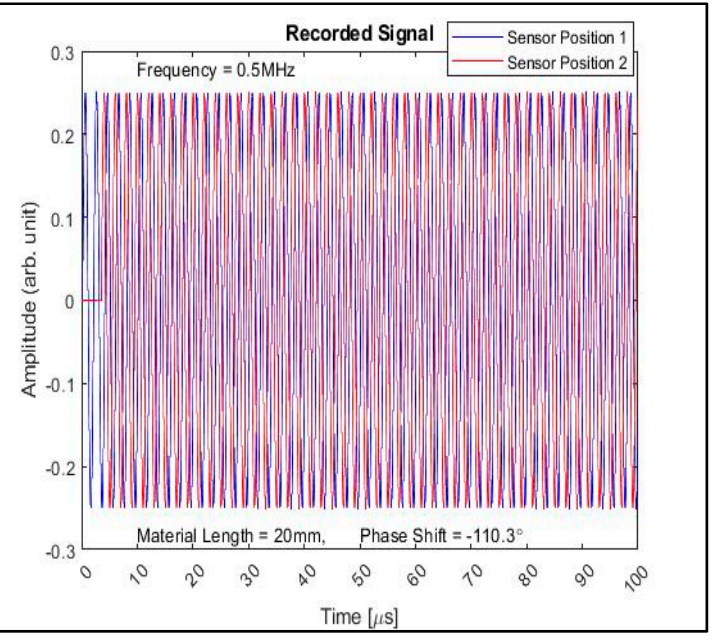

(b)

Figure 4. Two-frequency continuous wave method (TFcw) technique (a) 11.6 phase-shift at $0.6 \mathrm{MHz}$; (b) -110.3 phase-shift at $0.5 \mathrm{MHz}$. 
Table 3. Simulation results using multiple frequency continuous wave method (MFcw) technique.

\begin{tabular}{cccccc}
\hline $\begin{array}{c}\text { Temperature } \\
\left({ }^{\circ} \mathbf{C}\right)\end{array}$ & $\begin{array}{c}\text { Phase-Shift } \\
\left.\mathbf{( 0 . 5} \mathbf{~ M H z}) \mathbf{(}^{\circ}\right)\end{array}$ & $\begin{array}{c}\text { Phase-Shift } \\
\left.\mathbf{( 0 . 5 1 ~} \mathbf{M H z}) \mathbf{(}^{\circ}\right)\end{array}$ & $\begin{array}{c}\text { Phase-Shift } \\
\left.\mathbf{M H z}) \mathbf{(}^{\circ}\right)\end{array}$ & $\begin{array}{c}\text { Used } \\
\text { Ultrasonic } \\
\text { Speed (m/s) }\end{array}$ & $\begin{array}{c}\text { Calculated } \\
\text { Ultrasonic } \\
\text { Speed (m/s) }\end{array}$ \\
\hline 20 & -23.33 & 98.54 & -106.95 & 5904.88 & 5904.86 \\
20.1 & -23.261 & 98.61 & -105.64 & 5904.82 & 5904.8 \\
20.2 & -23.2 & 98.67 & -104.32 & 5904.75 & 5904.73 \\
20.3 & -23.13 & 98.74 & -103.01 & 5904.69 & 5904.67 \\
20.4 & -23.06 & 98.81 & -101.7 & 5904.63 & 5904.61 \\
20.5 & -23 & 98.88 & -100.38 & 5904.56 & 5904.54 \\
20.6 & -22.93 & 98.94 & -99.07 & 5904.5 & 5904.48 \\
20.7 & -22.87 & 99.01 & -97.76 & 5904.44 & 5904.41 \\
20.8 & -22.8 & 99.08 & -96.44 & 5904.37 & 5904.35 \\
20.9 & -22.74 & 99.14 & -95.13 & 5904.31 & 5904.29 \\
21 & -22.67 & 99.21 & -93.82 & 5904.24 & 5904.22 \\
\hline
\end{tabular}

The result of the simulation with $5 \mathrm{MHz}$ transducer and $15 \mathrm{~mm}$ steel plate is given in Figure 5 .

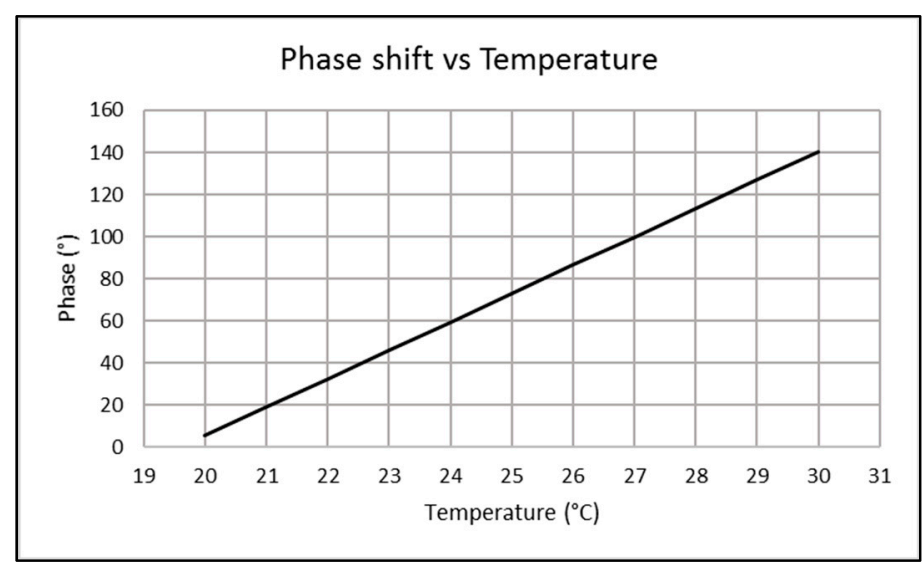

Figure 5. Phase-shift vs. temperature for $15 \mathrm{~mm}$ steel.

From the results of the simulations, both the pulse-echo method and the phase-shift method were able to resolve $0.1^{\circ} \mathrm{C}$ change in temperature. However, a pulser/receiver is needed to use the pulse-echo method. For a $0.1^{\circ} \mathrm{C}$ change detection, the pulser/receiver needs samples at up to $10 \mathrm{GHz}$ and the cost of such device can reach the $€ 20,000$ mark. However, for the phase-shift method, the cost of a phase detector is under $€ 400$. The phase-shift method was chosen because of its cost effectiveness, therefore, the experiments described in Section 3 are all based on the phase-shift method.

\section{Experiments}

\subsection{Experimental Setup}

Based on results from the k-Wave simulations, a phase-shift experiment was set up as shown in Figure 6. 


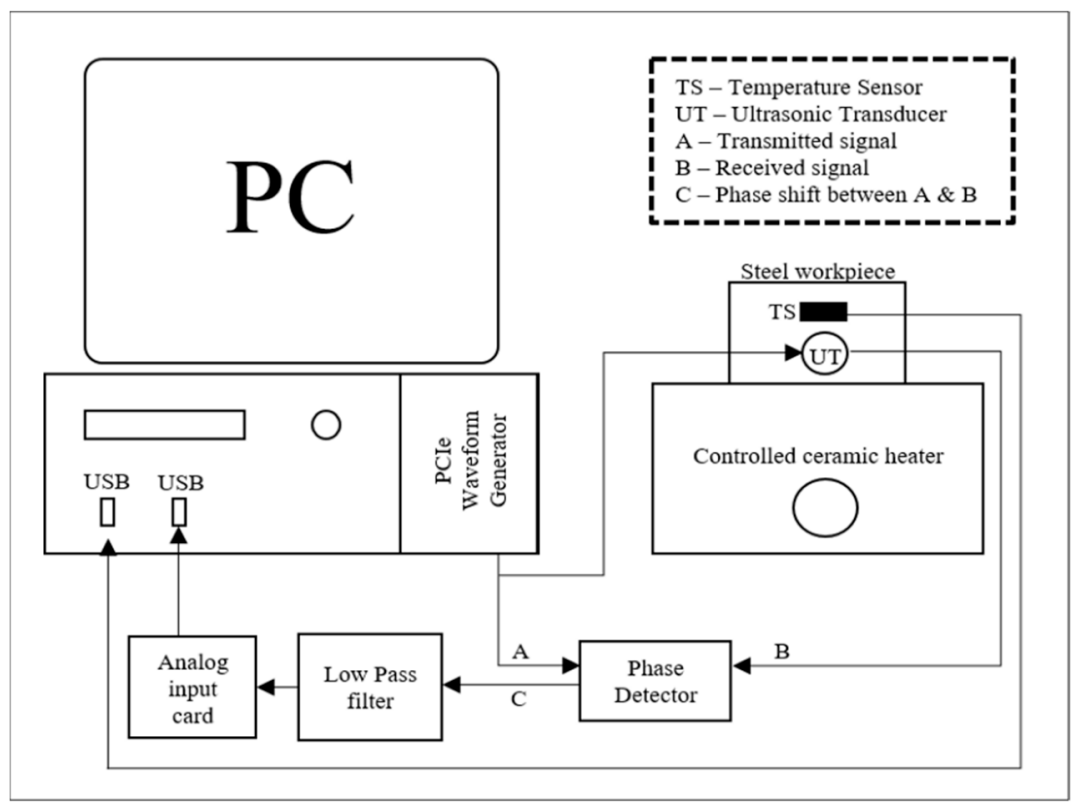

Figure 6. Ultrasonic phase-shift experimental setup.

A sinusoidal waveform was generated using a Spectrum M2i.6022-exp arbitrary waveform generator (Spectrum Instrumentation, Ahrensfelder, Grosshansdorf, Germany) . The waveform was then sent to the input A port of the Analog Devices AD8302 (Analog Devices, Norwood, MA, USA) phase detection board and the transmitter probe of a $5 \mathrm{MHz}$ ultrasonic transducer. Using steel (type EN24T) as the medium of propagation, the received signal from the receiver probe of the transducer was then sent to the input B port of the phase detector. The phase detector calculates the difference in phase between the transmitted and the received signal and sends the voltage equivalent as the phase difference value through the phase-out port of the phase detector board. To reduce noise from the 'phase' value, a low pass filter was used to cut off high frequency signals. A $3.4 \mathrm{~Hz}$ filter was used, however, a cut off frequency of similar value will give satisfactory results. An NI-9239 analogue input card (National Instruments, Austin, TX, USA) was used for data acquisition which was then stored using NI LabVIEW. A calibrated Maxim DS18B20 digital temperature sensor was used as reference and the measured temperature was recorded using WinTCal software, which is proprietary to the research group. A PT100 temperature sensor was used for monitoring finer resolution changes of $0.1^{\circ} \mathrm{C}$. Using a thermostatic temperature controller, a ceramic heater was used to gradually change the temperature of the steel workpiece in predefined steps. An ultrasonic transducer with a center frequency of $5 \mathrm{MHz}$ and approximate bandwidth of $3 \mathrm{MHz}$ was used. The effect of the change in temperature on the phase of the ultrasonic wave was recorded and will be discussed in the results section.

\subsection{Experimental Results}

Using the setup described in Section 3.1, the AD8302 board was used to obtain the voltage equivalent values of phase-shift. A typical curve showing the relationship between the phase output (V) and the equivalent phase difference (Degrees) is given in Figures 7 and 8, respectively. Figure 7 is the response specified in the datasheet while Figure 8 was obtained experimentally using the same parameters as those used for the phase-shift experiments $-2 \mathrm{~V}$ input, $40 \mathrm{mV}$ received signal and $5 \mathrm{MHz}$ ultrasonic frequency. 


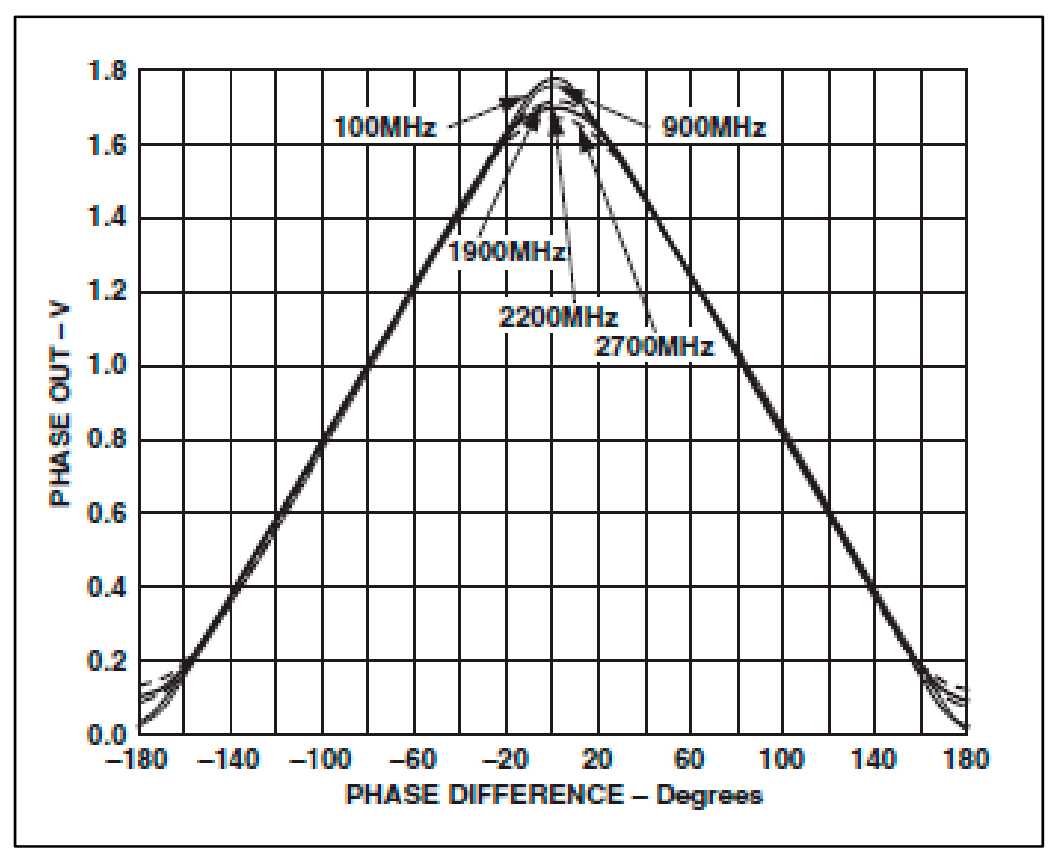

Figure 7. Phase Output vs. Input Phase Difference [23].

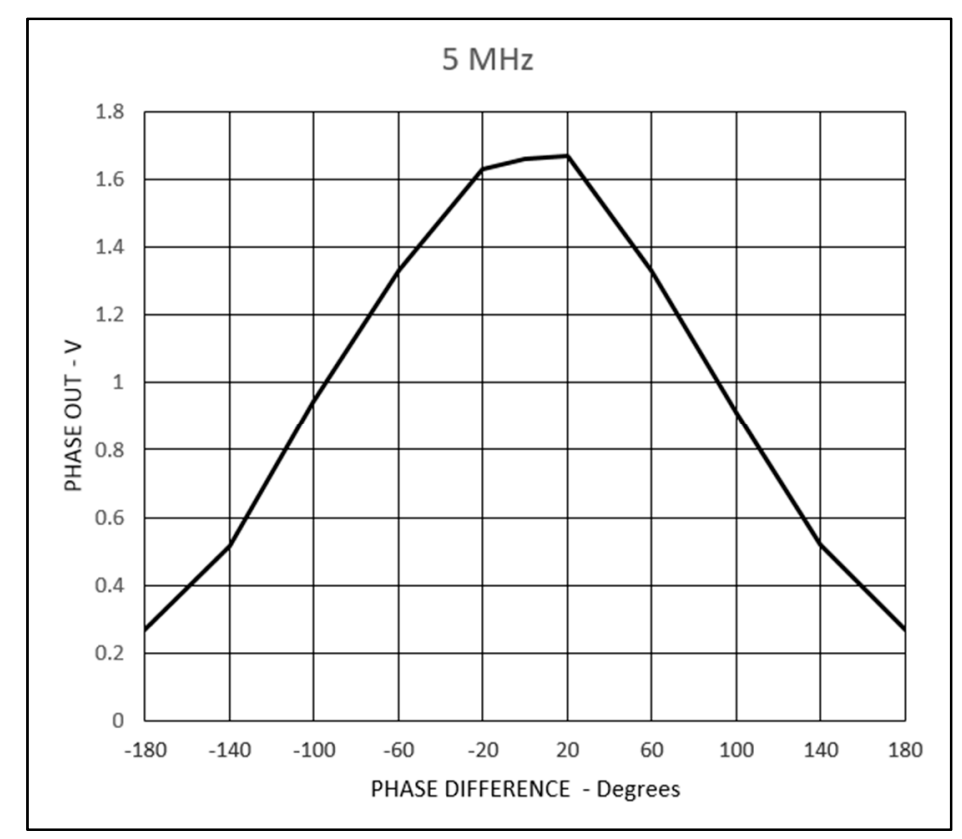

Figure 8. Phase Output vs. Input Phase Difference using the same parameters for the experiment.

Based on the phase-shift simulation, a steel sample of $15 \mathrm{~mm}$ (type EN24T) was used for the ultrasonic experiment. With a $5 \mathrm{MHz}$ transducer signal, measurements were made for a temperature range of 20 to $30^{\circ} \mathrm{C}$. The choice of this temperature range is based on a typical temperature variation during manufacturing processes. For the first experiment, temperature was varied in steps of $1{ }^{\circ} \mathrm{C}$ - thereafter, another experiment was carried out from the range of 21.5 to $23.5^{\circ} \mathrm{C}$ in $0.1^{\circ} \mathrm{C}$ steps. The 'Phase Out' values are the output from AD8032 phase detection board, which represents the phase-shift between the transmitted and the received ultrasonic signals. The recorded phase out vs. temperatures for both experiments are given in Figures 9 and 10. 


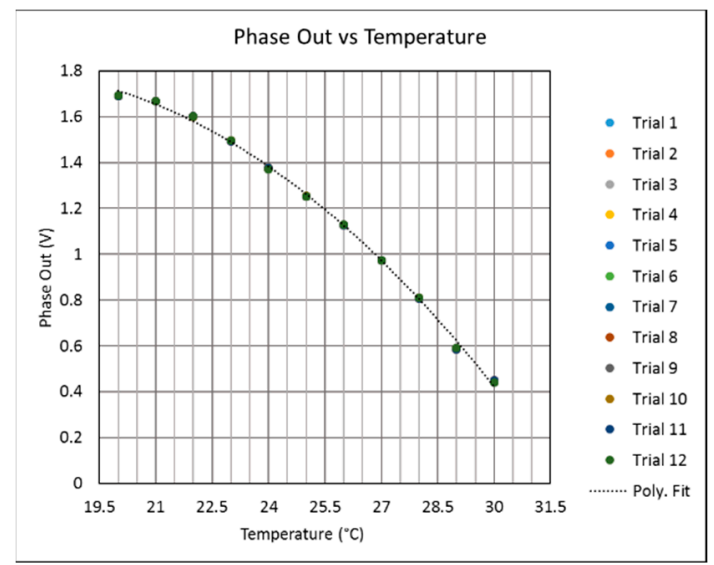

(a)

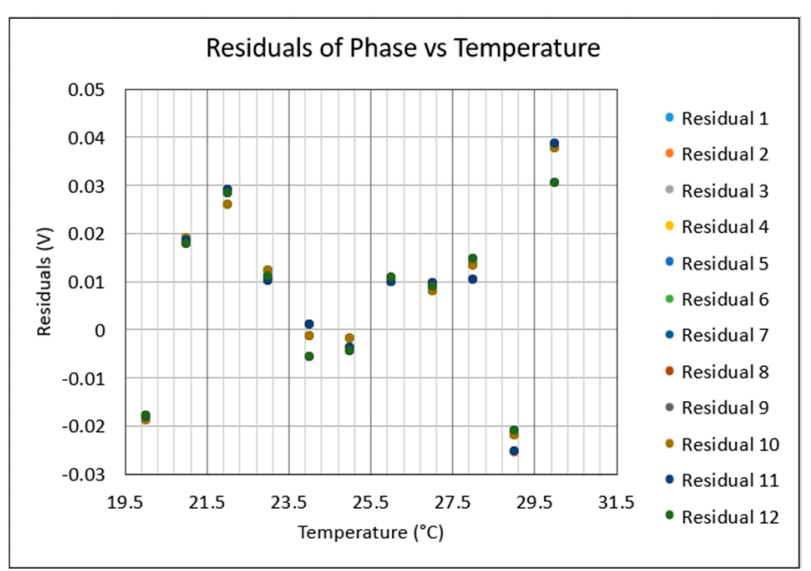

(b)

Figure 9. Results of 20 to $30^{\circ} \mathrm{C}$ range in $1^{\circ} \mathrm{C}$ steps (a) Phase output vs. temperature; (b) Residual plot.

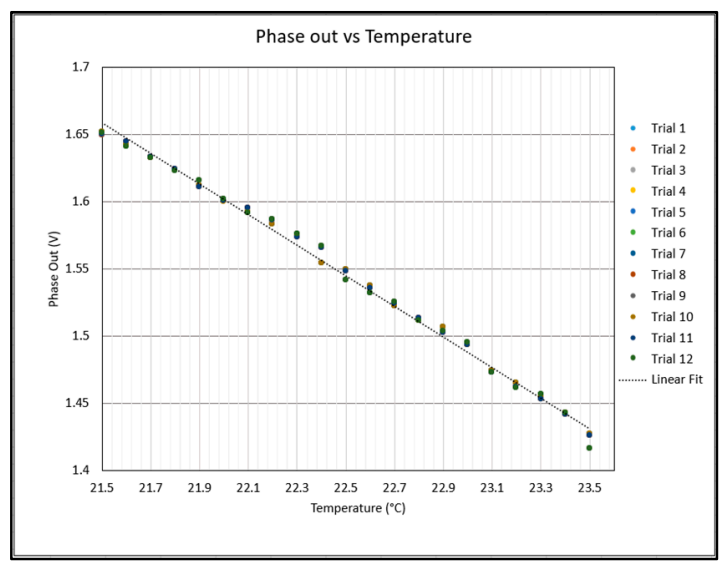

(a)

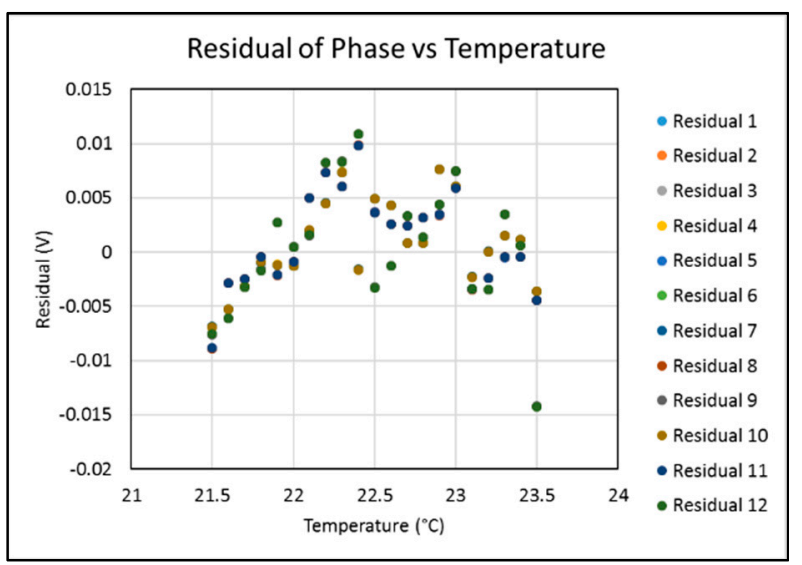

(b)

Figure 10. Results of 21.5 to $23.5^{\circ} \mathrm{C}$ range in $0.1^{\circ} \mathrm{C}$ steps (a) Phase output vs. temp; (b) Residual plot.

\section{Discussion}

The simulation, as well as experimental results, confirm that with the phase-shift technique, temperature change in a material can be measured, these results agree with the reviewed literature and other existing studies based on the dependence of ultrasonic velocity on temperature. The achievable measurement resolution as well as the range of measurement depend on the choice of frequency. Ultrasonic frequencies for phase-shift measurement need to be chosen to suit the expected range of measurement and the use of wideband transducers covering the expected range of measurement can reduce the cost of the test set up. For this experiment, where a $15 \mathrm{~mm}$ steel plate was used, the range can be increased with the selection of suitable frequencies based on Equations (3) and (5). Lower frequencies will result in a higher range, but with lower resolution. It is possible to use the TFcw method with low and high frequency transducers-this will improve both the resolution and the range. Two major limitations of the AD8302 board are the lack of clarity on the sign of the phase difference based on the reading obtained from the phase output in voltage and the non-linearity experienced at the extremes as shown in Figures 7 and 8. It is possible to avoid the non-linear regions by carefully selecting the transducer frequency for the length of the material whose temperature is to be measured. Changes in temperature can be measured reliably over the linear region, even when the sign of the phase is not known. 


\section{Conclusions}

This study showed that an ultrasonic measurement of the speed of sound in a metal based on the phase-shift method can be used to obtain the core temperature of the metal with a resolution of up to $0.1^{\circ} \mathrm{C}$. Based on simulation results of the two main ultrasonic measurement techniques-the pulse-echo and the phase-shift techniques-phase-shift is the less expensive technique for high resolution ultrasonic thermometry in metals. The two-frequency continuous wave method (TFCW) and multiple frequency continuous wave method (MFcw) are two improvements on the general phase-shift method for longer range and finer resolution measurements. A simulation was performed to observe the individual effects of expansion and change in ultrasonic velocity on time-of-flight. Based on the simulation, ultrasonic velocity can be relied upon for measuring time of flight and, where necessary, compensations can be made for the material expansion. Using a $5 \mathrm{MHz}$ transducer, $15 \mathrm{~mm}$ steel plate and varying the temperature from 20 to $30^{\circ} \mathrm{C}$, a voltage equivalent of phase difference was obtained. Overall, the results demonstrate that phase-shift ultrasonic thermometry can be used for core temperature measurement with a resolution of $0.1{ }^{\circ} \mathrm{C}$. A possible application of this study would be for temperature monitoring during co-ordinate metrology, such as on a co-ordinate measuring machine. As part of future work to deploy this setup in subtractive manufacturing, more experiments will be undertaken to understand the effects of swarf and coolant on ultrasonic thermometry. Future experiments will also be carried out to understand the effect of temperatures above $200^{\circ} \mathrm{C}$ on ultrasonic velocity. Also, as different materials have different physical properties, ultrasonic thermometry must be calibrated for the material of which the measurement is to be made. Future work will also address the possibility of using ultrasonic thermometry to measure the temperature of a region or a point within different materials. The use of switching algorithms to different frequencies for different materials and material sizes will also be researched.

Author Contributions: Formal analysis, O.F.O.; Funding acquisition, S.F.; Methodology, O.F.O. and S.F.; Software, O.F.O.; Supervision, S.F., A.P.L. and N.S.M.; Writing-original draft, O.F.O.; Writing-review and editing, O.F.O., S.F., A.P.L. and N.S.M.

Funding: This research was funded by the UK's Engineering and Physical Sciences Research Council (EPSRC), grant number EP/P006930/1.

Conflicts of Interest: The authors declare no conflicts of interest.

\section{References}

1. Cverna, F. ASM Ready Reference: Thermal Properties of Metals; ASM International: Geauga County, OH, USA, 2002.

2. da Bacci Silva, M.; Wallbank, J. Cutting temperature: Prediction and measurement methods-A review. J. Mater. Process. Technol. 1999, 88, 195-202. [CrossRef]

3. Olson, L.; Throne, R.; Rost, E. Improved Inverse Solutions for On-Line Machine Tool Monitoring. J. Manuf. Sci. Eng. 2004, 126, 311. [CrossRef]

4. Herbert, E.G. The measurement of cutting temperatures. Proc. Inst. Mech. Eng. 1926, 110, 289-329. [CrossRef]

5. Dhar, N.; Islam, S.; Kamruzzaman, M.; Ahmed, T. The Calibration of Tool-Work Thermocouple in Turning Steels. In Proceedings of the National Conference on Industrial Problems on Machines and Mechanisms (IPROMM-2005), Kharagpur, India, 24-25 February 2005.

6. Santos, M.C.; Araújo Filho, J.S.; Barrozo, M.A.S.; Jackson, M.J.; Machado, A.R. Development and application of a temperature measurement device using the tool-workpiece thermocouple method in turning at high cutting speeds. Int. J. Adv. Manuf. Technol. 2017, 89, 2287-2298. [CrossRef]

7. da Silva, M.B.; Wallbank, J. Temperature Measurements in Machining Using Infrared Sensor, COBEM97, Cong; Brasileiro de Engenharia Mecânica: Bauru, Brazil, 1997.

8. FLIR Systems Inc. FLIR E4. P/N 63901-0101. Available online: https://www.termokamery-flir.cz/wp-content/ uploads/2013/10/Datasheet-E4 (accessed on 3 September 2019).

9. Takahashi, M.; Ihara, I. Ultrasonic monitoring of internal temperature distribution in a heated material. Jpn. J. Appl. Phys. 2008, 47, 3894-3898. [CrossRef] 
10. Marioli, D.; Narduzzi, C.; Offelli, C.; Petri, D.; Sardini, E.; Taroni, A. Digital Time-of-Flight Measurement for Ultrasonic Sensors. IEEE Trans. Instrum. Meas. 1992, 41, 93-97. [CrossRef]

11. Gueuning, F.E.; Varlan, M.; Eugène, C.E.; Dupuis, P. Accurate distance measurement by an autonomous ultrasonic system combining time-of-flight and phase-shift methods. IEEE Trans. Instrum. Meas. 1997, 46, 1236-1240. [CrossRef]

12. Aldawi, F.; Longstaff, A.P.; Fletcher, S.; Mather, P.; Myers, A. A high accuracy ultrasound distance measurement system using binary frequency shift-keyed signal and phase detection. In School of Computing and Engineering Researchers' Conference, University of Huddersfield. 2007. Available online: http://eprints.hud. ac.uk/3789/1/17_-_Additional_Paper_1_F_Aldawi_FINAL.pdf (accessed on 3 September 2019).

13. Huang, C.F.; Young, M.S.; Li, Y.C. Multiple-frequency continuous wave ultrasonic system for accurate distance measurement. Rev. Sci. Instrum. 1999, 70, 1452-1458. [CrossRef]

14. Huang, K.-N.; Huang, Y.-P. Multiple-frequency ultrasonic distance measurement using direct digital frequency synthesizers. Sens. Actuators A Phys. 2009, 149, 42-50. [CrossRef]

15. Hu, B.; Zhu, Y.; Shi, C. Effect of Temperature on Solid Ultrasonic Propagation Using Finite Element Method and Experiments. In Proceedings of the 2018 IEEE Far East NDT New Technology \& Application Forum (FENDT), Fujian, China, 6-8 July 2018; pp. 107-111.

16. Ihara, I.; Kosugi, A. Noncontact Temperature Sensing of Heated Cylindrical End using Laser Ultrasonic Technique. In Proceedings of the 2017 Eleventh International Conference on Sensing Technology (ICST), Sydney, Australia, 4-6 April 2017; pp. 1-3.

17. Treeby, B.; Cox, B.; Jaros, J. k-Wave: A MATLAB Toolbox for the Time Domain Simulation of Acoustic Wave Fields-User Manual. Available online: http://www.k-wave.org/manual/k-wave_user_manual_1.1.pdf (accessed on 3 September 2019).

18. Barshan, B. Fast processing techniques for accurate ultrasonic range measurements. Meas. Sci. Technol. 2000, 11, 45-50. [CrossRef]

19. Huang, K.N.; Huang, C.F.; Li, Y.C.; Young, M.S. High precision, fast ultrasonic thermometer based on measurement of the speed of sound in air. Rev. Sci. Instrum. 2002, 73, 4022. [CrossRef]

20. Queirós, R.; Martins, R.C.; Girão, P.S.; Serra, A.C. A New Method for High Resolution Ultrasonic Ranging in Air. In Proceedings of the XVIII IMEKO World Congress, Rio de Janeiro, Brazil, 17-22 September 2006; pp. 1-4.

21. Tsai, W.Y.; Chen, H.C.; Liao, T.L. High accuracy ultrasonic air temperature measurement using multi-frequency continuous wave. Sens. Actuators A Phys. 2006, 132, 526-532. [CrossRef]

22. Ihara, I.; Takahashi, M. A novel ultrasonic thermometry for monitoring temperature profiles in materials. In Proceedings of the XIX IMEKO World Congress, Lisbon, Portugal, 6-11 September 2009; Volume 3, pp. 1635-1639.

23. Analog Devices. LF-2.7 GHz RF/IF Gain and Phase Detector AD8302. Available online: https: //www.analog.com/media/cn/technical-documentation/evaluation-documentation/AD8302.pdf (accessed on 3 September 2019).

(C) 2019 by the authors. Licensee MDPI, Basel, Switzerland. This article is an open access article distributed under the terms and conditions of the Creative Commons Attribution (CC BY) license (http://creativecommons.org/licenses/by/4.0/). 